\title{
A Viscosity Approximation Scheme for Finding Common Solutions of Mixed Equilibrium Problems, a Finite Family of Variational Inclusions, and Fixed Point Problems in Hilbert Spaces
}

\author{
Bin-chao Deng, ${ }^{1}$ Tong Chen, ${ }^{1}$ and Baogui Xin ${ }^{1,2}$ \\ ${ }^{1}$ School of Management, Tianjin University, Tianjin 300072, China \\ 2 School of Economics and Management, Shandong University of Science and Technology, \\ Qingdao 266510, China
}

Correspondence should be addressed to Bin-chao Deng, dbchao1985@yahoo.com.cn

Received 16 February 2012; Accepted 10 April 2012

Academic Editor: Yeong-Cheng Liou

Copyright (c) 2012 Bin-chao Deng et al. This is an open access article distributed under the Creative Commons Attribution License, which permits unrestricted use, distribution, and reproduction in any medium, provided the original work is properly cited.

\begin{abstract}
We introduce an iterative method for finding a common element of set of fixed points of nonexpansive mappings, the set of solutions of a finite family of variational inclusion with setvalued maximal monotone mappings and inverse strongly monotone mappings, and the set of solutions of a mixed equilibrium problem in Hilbert spaces. Under suitable conditions, some strong convergence theorems for approximating this common elements are proved. The results presented in the paper improve and extend the main results of Plubtemg and Sripard and many others.
\end{abstract}

\section{Introduction}

Let $H$ be a real Hilbert space whose inner product and norm are denoted by $\langle\cdot, \cdot\rangle$ and $\|\cdot\|$, respectively. Let $C$ be a nonempty closed convex subset of $H$, and let $F$ be a bifunction of $C \times C$ into $\mathbb{R}$ which is the set of real numbers. The equilibrium problem for $F: C \times C \rightarrow \mathbb{R}$ is to find $x \in C$ such that

$$
F(x, y) \geq 0, \quad \forall y \in C
$$

The set of solutions of (1.1) is denoted by $\operatorname{EP}(F)$. The mixed equilibrium problem for two bifunction of $F_{1}, F_{2}: C \times C \rightarrow \mathbb{R}$ is to find $x \in C$ such that

$$
F_{1}(x, y)+F_{2}(x, y)+\langle A x, x-y\rangle \geq 0, \quad \forall y \in C
$$


In the sequel we will indicate by $\operatorname{MEP}\left(F_{1}, F_{2}, A\right)$ the set of solution of our mixed equilibrium problem. If $A=0$ we denote $\operatorname{MEP}\left(F_{1}, F_{2}, 0\right)$ with $\operatorname{MEP}\left(F_{1}, F_{2}\right)$.

In 2005, Combettes and Hirstoaga [1] introduced an iterative scheme of finding the best approximation to the initial data when $\mathrm{EP}(F)$ is nonempty and proved a strong convergence theorem. Let $A: C \rightarrow H$ be a nonlinear mapping. The classical variational inequality which is denoted by $\operatorname{VI}(A, C)$ is to find $x \in C$ such that

$$
\langle A x, y-x\rangle \geq 0, \quad \forall y \in C
$$

The variational inequality has been extensively studied in the literature; see, for example, $[2,3]$ and the reference therein. Recall that mapping $T$ of $C$ into itself is called nonexpansive if

$$
\|T x-T y\| \leq\|x-y\|, \quad \forall x, y \in C \text {. }
$$

A mapping $f: C \rightarrow C$ is called contractive if there exists a constant $\beta \in(0,1)$ such that

$$
\|f x-f y\| \leq \beta\|x-y\|, \quad \forall x, y \in C
$$

We denote by $F_{i x}(T)$ the set of fixed points of $T$.

Some methods have been proposed to solve the equilibrium problem and fixed point problem of nonexpansive mapping; see, for instance, $[2,4-6]$ and the references therein. In 2007, Plubtieng and Punpaeng [6] introduced the following iterative scheme. Let $x_{1} \in H$ and let $\left\{x_{n}\right\}$ and $\left\{u_{n}\right\}$ be sequences generated by

$$
\begin{gathered}
F\left(u_{n}, y\right)+\frac{1}{r_{n}}\left\langle y-u_{n}, u_{n}-x_{n}\right\rangle \geq 0, \quad \forall y \in H, \\
x_{n+1}=\alpha_{n} \gamma f\left(x_{n}\right)+\left(I-\alpha_{n} A\right) T u_{n}, \quad \forall n \in \mathbb{N} .
\end{gathered}
$$

They proved that if the sequences $\left\{\alpha_{n}\right\}$ and $\left\{r_{n}\right\}$ of parameters satisfy appropriate conditions, then the sequences $\left\{x_{n}\right\}$ and $\left\{u_{n}\right\}$ both converge strongly to the unique solution of the variational inequality

$$
\langle(A-\gamma f) z, z-x\rangle \geq 0, \quad \forall x \in F_{i x}(T) \cap \operatorname{EP}(F),
$$

which is the optimality condition for the minimization problem

$$
\min _{x \in F_{i x}(T) \cap \mathrm{EP}(F)} \frac{1}{2}\langle A x, x\rangle-h(x)
$$

where $h$ is a potential function for $\gamma f$.

Let $A: H \rightarrow H$ be a single-valued nonlinear mapping, and let $M: H \rightarrow 2^{H}$ be a set-valued mapping. We consider the following variational inclusion, which is to find a point $u \in H$ such that

$$
\theta \in A(u)+M(u)
$$


where $\theta$ is the zero vector in $H$. The set of solutions of problem (1.9) is denoted by $I(A, M)$. Let $A_{i}: H \rightarrow H, i=1,2, \ldots N$ be single-valued nonlinear mappings, and let $M_{i}: H \rightarrow 2^{H}, i=1,2, \ldots N$, be set-valued mappings. If $A \equiv 0$, then problem (1.9) becomes the variational inclusion problem introduced by Rockafellar [7]. If $M=\partial \delta_{C}$, where $C$ is a nonempty closed convex subset of $H$ and $\delta_{C}: H \rightarrow[0, \infty]$ is the indicator function of $C$, that is,

$$
\delta_{C}(x)= \begin{cases}0, & x \in C, \\ +\infty, & x \notin C,\end{cases}
$$

then the variational inclusion problem (1.9) is equivalent to variational inequality problem (1.3). It is known that (1.9) provides a convenient framework for the unified study of optimal solutions in many optimization-related areas including mathematical programming, complementarity, variational inequalities, optimal control, mathematical economics, equilibria, and game theory. Also various types of variational inclusions problems have been extended and generalized (see [8] and the references therein). We introduce following a finite family of variational inclusions, which are to find a point $u \in H$ such that

$$
\theta \in A_{i}(u)+M_{i}(u), \quad i=1,2, \ldots N,
$$

where $\theta$ is the zero vector in $H$. The set of solutions of problem (1.11) is denoted by $\bigcap_{i=1}^{N} I\left(A_{i}, M_{i}\right)$. The formulation (1.11) extends this formalism to a finite family of variational inclusions covering, in particular, various forms of feasibility problems (see, e.g., [9]).

In 2009, Plubtemg and Sripard [10] introduced the following iterative scheme for finding a common element of set of solutions to the problem (1.9) with multivalued maximal monotone mapping and inverse-strongly monotone mapping, the set solutions of an equilibrium problem and the set of fixed points of a nonexpansive mapping in Hilbert spaces. Starting with an arbitrary $x_{1} \in H$, define sequence $\left\{x_{n}\right\},\left\{y_{n}\right\}$, and $\left\{u_{n}\right\}$ by

$$
\begin{gathered}
F\left(u_{n}, y\right)+\frac{1}{r_{n}}\left\langle y-u_{n}, u_{n}-x_{n}\right\rangle \geq 0, \quad \forall y \in H, \\
y_{n}=J_{M, \lambda}\left(u_{n}-\lambda A u_{n}\right), \quad \forall n>0, \\
x_{n+1}=\alpha_{n} \gamma f\left(x_{n}\right)+\left(I-\alpha_{n} B\right) S_{n} y_{n},
\end{gathered}
$$

for all $n \in N$, where $\lambda \in(0,2 \alpha],\left\{\alpha_{n}\right\} \subset[0,1]$, and $\left\{r_{n}\right\} \subset(0, \infty) ; B$ is a strongly positive bounded linear operator on $H$, and $\left\{S_{n}\right\}$ is a sequence of nonexpansive mappings on $H$. They proved that under certain appropriate conditions imposed on $\left\{\alpha_{n}\right\}$ and $\left\{r_{n}\right\}$, the sequence $\left\{x_{n}\right\},\left\{y_{n}\right\}$, and $\left\{u_{n}\right\}$ generated by (1.12) converge strongly to $z \in \bigcap_{i=1}^{\infty} F_{i x}\left(S_{i}\right) \cap I(A, M) \cap$ $\mathrm{EP}(F)$, where $z=P_{\bigcap_{i=1}^{\infty} F_{i x}\left(S_{i}\right) \cap I(A, M) \cap \mathrm{EP}(F)} f(z)$.

In 2011, Yao et al. [11] considered the following iterative method for finding a common element of set of solutions to the problem (1.9) with multi-valued maximal monotone mapping and inverse-strongly monotone mapping, the set solutions of a mixed equilibrium problem, and the set of fixed points of an infinite family of nonexpansive mappings in Hilbert spaces. Let $F: H \times H \rightarrow \mathbb{R}$ be a bifunction, $A$ be a strongly positive bounded linear operator, and $B_{1}, B_{2}: H \rightarrow H$ be inverse strongly monotone and let inverse strongly monotone, and 
$\varphi: H \rightarrow \mathbb{R}$ be a lower semicontinuous and convex function. For any initial $x_{0}$ is selected in $H$ arbitrarily

$$
\begin{gathered}
F\left(u_{n}, y\right)+\varphi(y)-\varphi\left(u_{n}\right)+\frac{1}{r}\left\langle y-u_{n}, u_{n}-\left(x_{n}-r B_{1} x_{n}\right)\right\rangle \geq 0, \quad \forall y \in H, \\
x_{n+1}=\alpha_{n} \gamma f\left(x_{n}\right)+\beta_{n} x_{n}+\left(\left(1-\beta_{n}\right) I-\alpha_{n} B\right) W_{n} J_{R, \lambda}\left(u_{n}-\lambda B_{2} u_{n}\right), \quad n \geq 0,
\end{gathered}
$$

where $\left\{\alpha_{n}\right\}$ and $\left\{\beta_{n}\right\}$ are two sequences in $[0,1]$ and $W_{n}$ is an infinite family of nonexpansive mappings. They study the strong convergence of the iterative algorithm (1.13).

Motivated and inspired by Saeidi [12], Aoyama et al. [13], Plubieng and Punpaeng [6], Plubtemg and Sripard [10], Peng et al. [14], and Yao et al. [11], we introduce an iterative scheme for finding a common element of the set of solutions of a finite family of variational inclusion problems (1.11) with multi-valued maximal monotone mappings and inversestrongly monotone mappings, the set of solutions of a mixed equilibrium problem, and the set of fixed points of nonexpansive mappings in Hilbert space. Starting with an arbitrary $x_{1} \in H$, define sequence $\left\{x_{n}\right\},\left\{y_{n}\right\}$ and $\left\{u_{n}\right\}$ by

$$
\begin{gathered}
F_{1}\left(u_{n}, y\right)+F_{2}\left(u_{n}, y\right)+\frac{1}{r_{n}}\left\langle y-u_{n}, u_{n}-x_{n}\right\rangle \geq 0, \quad \forall y \in H, \\
y_{n}=J_{M_{N}, \lambda_{N, n}}\left(I-\lambda_{N, n} A_{N}\right) \cdots J_{M_{1}, \lambda_{1, n}}\left(I-\lambda_{1, n} A_{1}\right) u_{n}, \\
x_{n+1}=\epsilon_{n} \gamma f\left(x_{n}\right)+\beta_{n} x_{n}+\left(\left(1-\beta_{n}\right) I-\epsilon_{n} B\right) S_{n} y_{n},
\end{gathered}
$$

for all $n \in N$, where $\lambda_{i, n} \in\left(0,2 \alpha_{i}\right], i \in\{1,2, \ldots, N\},\left\{\epsilon_{n}\right\} \subset[0,1]$, and $\left\{r_{n}\right\} \subset$ $(0, \infty), B$ is a strongly positive bounded linear operator on $H$, and $\left\{S_{n}\right\}$ is a sequence of nonexpansive mappings on $H$. Under suitable conditions, some strong convergence theorems for approximating to this common elements are proved. Our results extend and improve some corresponding results in $[10,11,14]$ and the references therein.

\section{Preliminaries}

This section collects some lemmas which will be used in the proofs for the main results in next section.

Let $H$ be a real Hilbert space with inner product $\langle\cdot, \cdot\rangle$ and norm $\|\cdot\|$, respectively. It is well known that for all $x, y \in H$ and $\lambda \in[0,1]$, there holds

$$
\|\lambda x+(1-\lambda) y\|^{2}=\lambda\|x\|^{2}+(1-\lambda)\|y\|^{2}-\lambda(1-\lambda)\|x-y\|^{2} .
$$

Let $C$ be a nonempty closed convex subset of $H$. Then, for any $x \in H$, there exists a unique nearest point of $C$, denoted by $P_{C} x$. such that $\left\|x-P_{C} x\right\| \leq\|x-y\|$ for all $y \in C$. Such a $P_{C}$ is called the metric projection from $H$ into $C$. We know that $P_{C}$ is nonexpansive. It is also known that $P_{C} x \in C$ and

$$
\left\langle x-P_{C} x, P_{C} x-z\right\rangle \geq 0, \quad \forall x \in H, z \in C .
$$


It is easy to see that (2.2) is equivalent to

$$
\|x-z\|^{2} \geq\left\|x-P_{C} x\right\|^{2}+\left\|P_{C} x-z\right\|^{2}, \quad \forall x \in H, z \in C .
$$

For solving the mixed equilibrium problem for a bifunction $F: C \times C \rightarrow \mathbb{R}$, let us assume that $F$ satisfies the following conditions:

(A1) $F(x, x)=0$ for all $x \in C$;

(A2) $F$ is monotone, that is, $F(x, y)+F(y, x) \leq 0$ for all $x, y \in C$;

(A3) for each $x, y, z \in C$,

$$
\lim _{t \rightarrow 0} F(t z+(1-t) x, y) \leq F(x, y)
$$

(A4) for each $x \in C, y \mapsto F(x, y)$ is convex and lower semicontinuous.

Lemma 2.1 (see [15]). Let $C$ be a convex closed subset of a Hilbert spaces $H$.

Let $F_{1}: C \times C \rightarrow R$ be a bifunction such that

(f1) $F_{1}(x, x)=0$ for all $x \in C$;

(f2) $F_{1}$ is monotone and upper hemicontinuous in the first variable;

(f3) $F_{1}$ is lower semicontinuous and convex in the second variable.

Let $F_{2}: C \times C \rightarrow R$ be a bifunction such that

(h1) $F_{2}(x, x)=0$ for all $x \in C$;

(h2) $F_{2}$ is monotone and weakly upper semicontinuous in the first variable;

(h3) $F_{2}$ is convex in the second variable.

Moreover let us suppose that

(H) for fixed $r>0$ and $x \in C$ there exists a bounded set $K \subset C$ and $a \in K$ such that for all $z \in C \backslash K,-F_{1}(a, z)+F_{2}(z, a)+(1 / r)\langle a-z, z-x\rangle<0$.

For $r>0$ and $x \in H$, let $T_{r}: H \rightarrow C$ be a mapping defined by

$$
T_{r}(x)=\left\{y \in C: F_{1}(z, y)+F_{1}(z, y)+\frac{1}{r}\langle y-z, z-x\rangle \geq 0, \forall z \in C\right\}
$$

Called resolvent of $F_{1}$ and $F_{2}$.

Then,

(1) $T_{r} x \neq \emptyset$;

(2) $T_{r}$ is a single value;

(3) $T_{r}$ is firmly nonexpansive;

(4) $\operatorname{MEP}\left(F_{1}, F_{2}\right)=F_{i x}\left(T_{r} x\right)$ and it is closed and convex. 
Recall that a mapping $A: H \rightarrow H$ is called $\alpha$-inverse-strongly monotone, if there exists a positive number $\alpha$ such that

$$
\langle A u-A v, u-v\rangle \geq \alpha\|A u-A v\|^{2}, \quad \forall u, v \in H .
$$

Let $I$ be the identity mapping on $H$. It is well known that if $A: H \rightarrow H$ is $\alpha$-inversestrongly monotone, then $A$ is $1 / \alpha$-Lipschitz continuous and monotone mapping. In addition, if $0<\lambda \leq 2 \alpha$, then $I-\lambda A$ is a nonexpansive mapping.

A set-valued $M: H \rightarrow 2^{H}$ is called monotone, if for all $x, y \in H, f \in M x$, and $g \in M y$ imply $\langle x-y, f-g\rangle \geq 0$. A monotone mapping $M: H \rightarrow 2^{H}$ is maximal if its graph $G(M):\{(x, f) \in H \times H \mid f \in M(x)\}$ of $M$ is not properly contained in the graph of any other monotone mapping. It is known that a monotone mapping $M$ is maximal if and only if for $(x, f) \in H \times H,\langle x-y, f-g\rangle \geq 0$ for every $(y, g) \in G(H)$ implies $f \in M x$.

Let the set-valued $M: H \rightarrow 2^{H}$ be maximal monotone. we define the resolvent operator $J_{M, \lambda}$ associated with $M$ and $\lambda$ as follows:

$$
J_{M, \lambda}(u)=(I+\lambda M)^{-1}(u), \quad \forall u \in H,
$$

where $\lambda$ is a positive number. It is worth mentioning that the resolvent operator $J_{M, \lambda}$ is singlevalued, nonexpansive, and 1-inverse-strongly monotone, see for example [16] and that a solution of problem (1.9) is a fixed point of the operator $J_{M, \lambda}(I-\lambda A)$ for all $\lambda>0$, see for instance, [17]. Furthermore, a solution of a finite family of variational inclusion problems (1.11) is a common fixed point of $J_{M_{k}, \lambda}\left(I-\lambda A_{k}\right), k \in\{1, \ldots, N\}, \lambda>0$.

Lemma 2.2 (see [16]). Let $M: H \rightarrow 2^{H}$ be a maximal monotone mapping and $A: H \rightarrow H a$ Lipschitz-continuous mapping. Then the mapping $S=M+A: H \rightarrow 2^{H}$ is a maximal monotone mapping.

Lemma 2.3 (see [18]). Let $H$ be a Hilbert space, $C$ a nonempty closed subset of $H, f: H \rightarrow H$ a contraction with coefficient $0<\alpha<1$, and $B$ a strongly positive linear bounded operator with coefficient $\bar{\gamma}>0$. Then,

(1) if $0<\gamma<\bar{\gamma} / \alpha$, then $\langle x-y,(B-\gamma f) x-(B-\gamma f) y\rangle \geq(\bar{\gamma}-\gamma \alpha)\|x-y\|^{2}, x, y \in H$;

(2) if $0<\rho<\|B\|^{-1}$, then $\|I-\rho B\| \leq 1-\rho \bar{\gamma}$.

Lemma 2.4. For all $x, y \in H$, there holds the inequality

$$
\|x+y\|^{2} \leq\|x\|^{2}+2\langle y, x+y\rangle .
$$

Lemma 2.5 (the resolvent identity). Let $E$ be a Banach space, for $\lambda>0, \mu>0$ and $x \in E$,

$$
J_{\lambda} x=J_{\mu}\left(\frac{\mu}{\lambda} x+\left(1-\frac{\mu}{\lambda}\right) J_{\lambda} x\right)
$$


Lemma 2.6. Let $H$ be a Hilbert space. Let $A_{i}: H \rightarrow H, i=1,2, \ldots, N$ be $\alpha_{i}$-inverse-strongly monotone mappings, $M_{i}: H \rightarrow 2^{H}, i=1,2, \ldots, N$ maximal monotone mappings, and $\left\{\omega_{n}\right\}$ be a bounded sequence in $H$. Assume $\lambda_{j, n}>0, j=1,2, \ldots, N$, satisfy

(H1) $\lim _{n \rightarrow \infty} \sum_{n=1}^{\infty}\left|\lambda_{j, n}-\lambda_{j, n+1}\right|<\infty$,

(H2) $\lim \inf _{n \rightarrow \infty} \lambda_{j, n}>0$.

Set $\Theta_{n}^{k}=J_{M_{k}, \lambda_{k, n}}\left(I-\lambda_{k, n} A_{k}\right) \cdots J_{M_{1}, \lambda_{1, n}}\left(I-\lambda_{1, n} A_{1}\right)$ for $k \in\{1,2, \ldots, N\}$ and $\Theta_{n}^{0}=I$ for all $n$. Then, for $k \in\{1,2, \ldots, N\}$,

$$
\sum_{i=1}^{\infty}\left\|\Theta_{i+1}^{k} \omega_{i}-\Theta_{i}^{k} \omega_{i}\right\|<\infty
$$

Proof. From Lemma 2.5, we have, for all $k \in\{1,2, \ldots, N\}$,

$$
\begin{aligned}
& \left\|J_{M_{k}, \lambda_{k, n+1}}\left(I-\lambda_{k, n+1} A_{k}\right) \omega_{n}-J_{M_{k}, \lambda_{k, n}}\left(I-\lambda_{k, n} A_{k}\right) \omega_{n}\right\| \\
& \quad \leq\left|1-\frac{\lambda_{k, n}}{\lambda_{k, n+1}}\right|\left(\left\|J_{M_{k}, \lambda_{k, n+1}}\left(I-\lambda_{k, n+1} A_{k}\right) \omega_{n}\right\|+\left\|\omega_{n}\right\|\right) .
\end{aligned}
$$

Furthermore, from the definition of $\Theta_{n}^{k}$, it follows

$$
\Theta_{n}^{k}=J_{M_{k}, \lambda_{k, n}}\left(I-\lambda_{k, n} A_{k}\right) \Theta_{n}^{k-1}
$$

Combining (2.11) and (2.12), we obtain

$$
\begin{aligned}
\left\|\Theta_{n+1}^{k} \omega_{n}-\Theta_{n}^{k} \omega_{n}\right\| \leq & \left\|J_{M_{k}, \lambda_{k, n+1}}\left(I-\lambda_{k, n+1} A_{k}\right) \Theta_{n+1}^{k-1} \omega_{n}-J_{M_{k}, \lambda_{k, n}}\left(I-\lambda_{k, n} A_{k}\right) \Theta_{n}^{k-1} \omega_{n}\right\| \\
\leq & \left\|J_{M_{k}, \lambda_{k, n+1}}\left(I-\lambda_{k, n+1} A_{k}\right) \Theta_{n+1}^{k-1} \omega_{n}-J_{M_{k}, \lambda_{k, n+1}}\left(I-\lambda_{k, n+1} A_{k}\right) \Theta_{n}^{k-1} \omega_{n}\right\| \\
& +\left\|J_{M_{k}, \lambda_{k, n+1}}\left(I-\lambda_{k, n+1} A_{k}\right) \Theta_{n}^{k-1} \omega_{n}-J_{M_{k}, \lambda_{k, n}}\left(I-\lambda_{k, n} A_{k}\right) \Theta_{n}^{k-1} \omega_{n}\right\| \\
\leq & \left\|\Theta_{n+1}^{k-1} \omega_{n}-\Theta_{n}^{k-1} \omega_{n}\right\|+\left|1-\frac{\lambda_{k, n}}{\lambda_{k, n+1}}\right| \\
& \times\left(\left\|J_{M_{k}, \lambda_{k, n+1}}\left(I-\lambda_{k, n+1} A_{k}\right) \Theta_{n}^{k-1} \omega_{n}\right\|+\left\|\omega_{n}\right\|\right) \\
\leq & \left\|\Theta_{n+1}^{k-1} \omega_{n}-\Theta_{n}^{k-1} \omega_{n}\right\|+\left|1-\frac{\lambda_{k, n}}{\lambda_{k, n+1}}\right| M_{1} \\
\leq & \cdots \\
\leq & \left\|\Theta_{n+1}^{0} \omega_{n}-\Theta_{n}^{0} \omega_{n}\right\|+\sum_{l=1}^{k}\left|1-\frac{\lambda_{l, n}}{\lambda_{l, n+1}}\right| M_{1} \\
= & \sum_{l=1}^{k}\left|1-\frac{\lambda_{l, n}}{\lambda_{l, n+1}}\right| M_{1},
\end{aligned}
$$

where $M_{1}=\sup \left\{\left\|\omega_{n}\right\|+\sum_{k=1}^{N}\left\|J_{M_{k}, \lambda_{k, n+1}}\left(I-\lambda_{k, n+1} A_{k}\right) \Theta_{n}^{k-1} \omega_{n}\right\|\right\}$. According to (H1) and (H2), then (2.11) holds. 
Lemma 2.7 (see [19]). Let $\left\{x_{n}\right\}$ and $\left\{z_{n}\right\}$ be bounded sequences in a Banach space $X$, and let $\left\{\beta_{n}\right\}$ be a sequence in $[0,1]$ with $0<\liminf _{n \rightarrow \infty} \beta_{n}$ and $\lim \sup _{n \rightarrow \infty} \beta_{n}<1$. Suppose

$$
x_{n+1}=\beta_{n} x_{n}+\left(1-\beta_{n}\right) z_{n}
$$

for all $n \geq 0$ and

$$
\limsup _{n \rightarrow \infty}\left(\left\|z_{n+1}-z_{n}\right\|-\left\|x_{n+1}-x_{n}\right\|\right) \leq 0
$$

Then $\lim _{n \rightarrow \infty}\left\|x_{n}-z_{n}\right\|=0$.

Lemma 2.8 (see [20]). Assume $\left\{\alpha_{n}\right\}$ is a sequence of nonnegative real numbers such that

$$
\alpha_{n+1} \leq\left(1-\gamma_{n}\right) \alpha_{n}+\delta_{n}, \quad n \geq 0,
$$

where $\left\{\alpha_{n}\right\}$ is a sequence in $(0,1)$ and $\left\{\delta_{n}\right\}$ is a sequence in $\mathbb{R}$ such that

(i) $\sum_{n=1}^{\infty} \gamma_{n}=\infty$,

(ii) $\lim \sup _{n \rightarrow \infty}\left(\delta_{n} / \gamma_{n}\right) \leq 0$ or $\sum_{n=1}^{\infty}\left|\delta_{n}\right|<\infty$.

Then $\lim _{n \rightarrow \infty} \alpha_{n}=0$.

\section{Main Results}

Theorem 3.1. Let $H$ be a real Hilbert space, let $F_{1}$ and $F_{2}$ be bifunction $H \times H \rightarrow \mathbb{R}$ satisfying (A1)(A4), and let $\left\{S_{n}\right\}$ be a sequence of nonexpansive mappings on $H$. Let $A_{i}: H \rightarrow H, i=1,2, \ldots, N$, be $\alpha_{i}$-inverse-strongly monotone mappings, and let $M_{i}: H \rightarrow 2^{H}, i=1,2, \ldots, N$ be maximal monotone mappings such that $\Omega:=\left(\bigcap_{n=1}^{\infty} F_{i x}\left(S_{n}\right)\right) \cap \operatorname{MEP}\left(F_{1}, F_{2}\right) \cap\left(\bigcap_{i=1}^{N} I\left(A_{i}, M_{i}\right)\right) \neq \emptyset$. Let $f$ be a contraction of $H$ into itself with a constant $\alpha \in(0,1)$, and let $B$ be a strongly positive bounded linear operator on $H$ with coefficient $\bar{\gamma}>0$ and $0<\gamma<\bar{\gamma} / \alpha$. Let $\left\{x_{n}\right\},\left\{y_{n}\right\}$, and $\left\{u_{n}\right\}$ be sequences generated by $x_{1} \in H$ and

$$
\begin{array}{r}
F_{1}\left(u_{n}, y\right)+F_{2}\left(u_{n}, y\right)+\frac{1}{r_{n}}\left\langle y-u_{n}, u_{n}-x_{n}\right\rangle \geq 0, \quad \forall y \in H, \\
y_{n}=J_{M_{N}, \lambda_{N, n}}\left(I-\lambda_{N, n} A_{N}\right) \cdots J_{M_{1}, \lambda_{1, n}}\left(I-\lambda_{1, n} A_{1}\right) u_{n}, \\
x_{n+1}=\epsilon_{n} \gamma f\left(x_{n}\right)+\beta_{n} x_{n}+\left(\left(1-\beta_{n}\right) I-\epsilon_{n} B\right) S_{n} y_{n},
\end{array}
$$

for all $n \in \mathbb{N}$, where $\beta_{n} \in(0,1), \lambda_{i, n} \in\left(0,2 \alpha_{i}\right], i \in\{1,2, \ldots, N\}$, satisfy (H1)-(H2) and $\left\{\epsilon_{n}\right\} \subset[0,1]$ and $\left\{r_{n}\right\} \subset(0, \infty)$ satisfy

(C1) $\lim _{n \rightarrow \infty} \epsilon_{n}=0$;

(C2) $\sum_{n=1}^{\infty} \epsilon_{n}=\infty$;

(C3) $\sum_{n=1}^{\infty}\left|\epsilon_{n+1}-\epsilon_{n}\right|<\infty$;

(C4) $\lim \inf _{n \rightarrow \infty} r_{n}>0$; 
Journal of Applied Mathematics

(C5) $\sum_{n=1}^{\infty}\left|r_{n+1}-r_{n}\right|<\infty$;

(C6) $\lim _{n \rightarrow \infty} \beta_{n}=0$.

Suppose that $\sum_{n=1}^{\infty} \sup \left\{\left\|S_{n+1} z-S_{n} z\right\|: z \in K\right\}<\infty$ for any bounded subset $K$ of $H$. Let $S$ be a mapping of $H$ into itself defined by $S x=\lim _{n \rightarrow \infty} S_{n} x$, for all $x \in H$ and suppose that $F_{i x}(S)=$ $\bigcap_{n=1}^{\infty} F_{i x}\left(S_{n}\right)$. Then, $\left\{x_{n}\right\},\left\{y_{n}\right\}$, and $\left\{u_{n}\right\}$ converge strongly to $z$, where $z=P_{\Omega}(I-B+\gamma f)(z)$ is $a$ unique solution of the variational inequality

$$
\langle(B-\gamma f) z, z-x\rangle \leq 0 \quad x \in \Omega .
$$

Proof. Since $\epsilon_{n} \rightarrow 0$, we will assume that $\epsilon_{n} \leq\left(1-\beta_{n}\right)\|B\|^{-1}$ and $1-\epsilon_{n}(\bar{\gamma}-\alpha \gamma)>0$. Observe that, if $\|u\|=1$, then

$$
\left\langle\left(\left(1-\beta_{n}\right) I-\epsilon_{n} B\right) u, u\right\rangle=\left(1-\beta_{n}\right)-\epsilon_{n}\langle B u, u\rangle \geq\left(1-\beta_{n}-\epsilon_{n}\|B\|\right) \geq 0 .
$$

By Lemma 2.3 we have

$$
\left\|\left(1-\beta_{n}\right) I-\epsilon_{n} B\right\| \leq\left(1-\beta_{n}\right)-\epsilon_{n} \bar{\gamma}
$$

Moreover, using the definition of $\Theta_{n}^{k}$ in Lemma 2.6, we have $y_{n}=\Theta_{n}^{N} u_{n}$. We divide the proof into several steps.

Step 1. The sequence $\left\{x_{n}\right\}$ is bounded.

Since $\epsilon_{n} \rightarrow 0$, we may assume that $\epsilon_{n} \leq\|B\|^{-1}$ for all $n$. Let $p \in \Omega$. Using the fact that $J_{M_{k}, \lambda_{k, n}}\left(I-\lambda_{k, n} A_{k}\right), k \in\{1,2, \ldots, N\}$, is nonexpansive and $p=J_{M_{k}, \lambda_{k, n}}\left(I-\lambda_{k, n} A_{k}\right) p$, we have

$$
\left\|y_{n}-p\right\|=\left\|\Theta_{n}^{N} u_{n}-\Theta_{n}^{N} p\right\| \leq\left\|u_{n}-p\right\| \leq\left\|T_{r} x_{n}-T_{r} p\right\| \leq\left\|x_{n}-p\right\|,
$$

for all $n \geq 1$. Then, we have

$$
\begin{aligned}
\left\|x_{n+1}-p\right\|= & \|\left(\left(1-\beta_{n}\right) I-\epsilon_{n} B\right)\left(S_{n} \Theta_{n}^{N} u_{n}-S_{n} \Theta_{n}^{N} p\right)+\epsilon_{n}\left(f\left(x_{n}\right)-f(p)\right) \\
& +\epsilon_{n}(\gamma f(p)-B p)+\beta_{n}\left(x_{n}-p\right) \| \\
\leq & \left(1-\epsilon_{n}(\bar{\gamma}-\alpha \gamma)\right)\left\|x_{n}-p\right\|+\epsilon_{n}(\bar{\gamma}-\alpha \gamma) \frac{\|\gamma f(p)-B p\|}{\bar{\gamma}-\alpha \gamma} .
\end{aligned}
$$

It follow from (3.6) and induction that

$$
\left\|x_{n}-p\right\| \leq \max \left\{\left\|x_{1}-p\right\|, \frac{\|\gamma f(p)-A p\|}{\bar{\gamma}-\alpha \gamma}\right\}, \quad \forall n \geq 1
$$

Hence $\left\{x_{n}\right\}$ is bounded and therefore $\left\{u_{n}\right\},\left\{y_{n}\right\},\left\{f\left(x_{n}\right)\right\}$, and $\left\{S_{n} y_{n}\right\}$ are also bounded. 
Step 2. We show that $\left\|x_{n+1}-x_{n}\right\| \rightarrow 0$.

Define $x_{n+1}=\beta_{n} x_{n}+\left(1-\beta_{n}\right) v_{n}$ for each $n \geq 0$. From the definition of $v_{n}$, we obtain

$$
\begin{aligned}
v_{n+1}-v_{n}= & \frac{1}{1-\beta_{n+1}}\left(x_{n+2}-\beta_{n+1} x_{n+1}\right)-\frac{1}{1-\beta_{n}}\left(x_{n+1}-\beta_{n} x_{n}\right) \\
= & \frac{\epsilon_{n+1} \gamma f\left(x_{n+1}\right)+\left(\left(1-\beta_{n+1}\right) I-\epsilon_{n+1} B\right) S_{n+1} y_{n+1}}{1-\beta_{n+1}}-\frac{\epsilon_{n} \gamma f\left(x_{n}\right)+\left(\left(1-\beta_{n}\right) I-\epsilon_{n} B\right) S_{n} y_{n}}{1-\beta_{n}} \\
= & \frac{\epsilon_{n+1} \gamma f\left(x_{n+1}\right)}{1-\beta_{n+1}}-\frac{\epsilon_{n} \gamma f\left(x_{n}\right)}{1-\beta_{n}}+S_{n+1} y_{n+1}-S_{n} y_{n}+\frac{\epsilon_{n}}{1-\beta_{n}} B S_{n} y_{n}-\frac{\epsilon_{n+1}}{1-\beta_{n+1}} B S_{n+1} y_{n+1} \\
= & \frac{\epsilon_{n+1}}{1-\beta_{n+1}}\left(\gamma f\left(x_{n+1}\right)-B S_{n+1} y_{n+1}\right)+\frac{\epsilon_{n}}{1-\beta_{n}}\left(\gamma f\left(x_{n}\right)-B S_{n} y_{n}\right) \\
& +S_{n+1} y_{n+1}-S_{n+1} y_{n}+S_{n+1} y_{n}-S_{n} y_{n} .
\end{aligned}
$$

It follows that

$$
\begin{aligned}
\left\|v_{n+1}-v_{n}\right\|-\left\|x_{n+1}-x_{n}\right\| \leq & \frac{\epsilon_{n+1}}{1-\beta_{n+1}}\left(\left\|\gamma f\left(x_{n+1}\right)\right\|+\left\|B S_{n+1} y_{n+1}\right\|\right) \\
& +\frac{\epsilon_{n}}{1-\beta_{n}}\left(\left\|\gamma f\left(x_{n}\right)\right\|+\left\|B S_{n} y_{n}\right\|\right)+\left\|S_{n+1} y_{n+1}-S_{n+1} y_{n}\right\| \\
& +\left\|S_{n+1} y_{n}-S_{n} y_{n}\right\|-\left\|x_{n+1}-x_{n}\right\| \\
\leq & \frac{\epsilon_{n+1}}{1-\beta_{n+1}}\left(\left\|\gamma f\left(x_{n+1}\right)\right\|+\left\|B S_{n+1} y_{n+1}\right\|\right) \\
& +\frac{\epsilon_{n}}{1-\beta_{n}}\left(\left\|\gamma f\left(x_{n}\right)\right\|+\left\|B S_{n} y_{n}\right\|\right)+\left\|y_{n+1}-y_{n}\right\| \\
& +\left\|S_{n+1} y_{n}-S_{n} y_{n}\right\|-\left\|x_{n+1}-x_{n}\right\| .
\end{aligned}
$$

By the suppose of $\sum_{n=1}^{\infty} \sup \left\{\left\|S_{n+1} z-S_{n} z\right\|: z \in K\right\}<\infty$, we obtain

$$
\lim _{n \rightarrow \infty}\left\|S_{n+1} y_{n}-S_{n} y_{n}\right\|=0
$$

From Lemma 2.6, we obtain

$$
\lim _{n \rightarrow \infty}\left\|\Theta_{n+1}^{N} u_{n+1}-\Theta_{n}^{N} u_{n+1}\right\|=0 .
$$

By $\Theta_{n}^{N}$ and $T_{r}$ being nonexpansive, we have

$$
\begin{aligned}
\left\|y_{n+1}-y_{n}\right\| & =\left\|\Theta_{n+1}^{N} u_{n+1}-\Theta_{n}^{N} u_{n}\right\| \\
& \leq\left\|\Theta_{n+1}^{N} u_{n+1}-\Theta_{n}^{N} u_{n+1}\right\|+\left\|\Theta_{n}^{N} u_{n+1}-\Theta_{n}^{N} u_{n}\right\|
\end{aligned}
$$


Journal of Applied Mathematics

$$
\begin{aligned}
& \leq\left\|\Theta_{n+1}^{N} u_{n+1}-\Theta_{n}^{N} u_{n+1}\right\|+\left\|u_{n+1}-u_{n}\right\| \\
& \leq\left\|\Theta_{n+1}^{N} u_{n+1}-\Theta_{n}^{N} u_{n+1}\right\|+\left\|T_{r} x_{n+1}-T_{r} x_{n}\right\| \\
& \leq\left\|\Theta_{n+1}^{N} u_{n+1}-\Theta_{n}^{N} u_{n+1}\right\|+\left\|x_{n+1}-x_{n}\right\| .
\end{aligned}
$$

Substituting (3.12) into (3.9), we get

$$
\begin{aligned}
\left\|v_{n+1}-v_{n}\right\|-\left\|x_{n+1}-x_{n}\right\| \leq & \frac{\epsilon_{n+1}}{1-\beta_{n+1}}\left(\left\|\gamma f\left(x_{n+1}\right)\right\|+\left\|B S_{n+1} y_{n+1}\right\|\right) \\
& +\frac{\epsilon_{n}}{1-\beta_{n}}\left(\left\|\gamma f\left(x_{n}\right)\right\|+\left\|B S_{n} y_{n}\right\|\right)+\left\|\Theta_{n+1}^{N} u_{n+1}-\Theta_{n}^{N} u_{n+1}\right\| \\
& +\left\|S_{n+1} y_{n}-S_{n} y_{n}\right\| .
\end{aligned}
$$

By (3.10), (3.11), and the conditions (C1) and (C6), we imply that

$$
\lim _{n \rightarrow \infty}\left(\left\|v_{n+1}-v_{n}\right\|-\left\|x_{n+1}-x_{n}\right\|\right)=0 .
$$

Hence, by Lemma 2.7, we have $\lim _{n \rightarrow \infty}\left\|v_{n}-x_{n}\right\|=0$. Consequently, it follows that

$$
\lim _{n \rightarrow \infty}\left\|x_{n+1}-x_{n}\right\|=\lim _{n \rightarrow \infty}\left(1-\beta_{n}\right)\left\|v_{n}-x_{n}\right\|=0 .
$$

From (3.11), (3.12), and (3.15), we also imply that

$$
\lim _{n \rightarrow \infty}\left\|y_{n+1}-y_{n}\right\|=0
$$

Step 3. We now show that

$$
\lim _{n \rightarrow \infty}\left\|\Theta_{n}^{k} u_{n}-\Theta_{n}^{k-1} u_{n}\right\|=0, \quad k=1,2, \ldots, N
$$

Indeed, let $p \in \Omega$. It follows from the firmly nonexpansiveness of $J_{M_{k}, \lambda_{k, n}}\left(I-\lambda_{k, n} A_{k}\right)$ that

$$
\begin{aligned}
\left\|\Theta_{n}^{k} u_{n}-p\right\|^{2} & =\left\|J_{M_{k}, \lambda_{k, n}}\left(I-\lambda_{k, n} A_{k}\right) \Theta_{n}^{k-1} u_{n}-J_{M_{k}, \lambda_{k, n}}\left(I-\lambda_{k, n} A_{k}\right) p\right\|^{2} \\
& \leq\left\langle\Theta_{n}^{k} u_{n}-p, \Theta_{n}^{k-1} u_{n}-p\right\rangle \\
& =\frac{1}{2}\left(\left\|\Theta_{n}^{k} u_{n}-p\right\|^{2}+\left\|\Theta_{n}^{k-1} u_{n}-p\right\|^{2}\left\|\Theta_{n}^{k} u_{n}-\Theta_{n}^{k-1} u_{n}\right\|^{2}\right),
\end{aligned}
$$

for each $k \in\{1,2, \ldots, N\}$. Thus we get

$$
\left\|\Theta_{n}^{k} u_{n}-p\right\|^{2} \leq\left\|\Theta_{n}^{k-1} u_{n}-p\right\|^{2}-\left\|\Theta_{n}^{k} u_{n}-\Theta_{n}^{k-1} u_{n}\right\|^{2},
$$


which implies that, for each $k \in\{1,2, \ldots, N\}$,

$$
\begin{aligned}
\left\|y_{n}-p\right\|^{2} & =\left\|\Theta_{n}^{N} u_{n}-p\right\|^{2} \leq\left\|\Theta_{n}^{0} u_{n}-p\right\|^{2}-\sum_{k=1}^{N}\left\|\Theta_{n}^{k} u_{n}-\Theta_{n}^{k-1} u_{n}\right\|^{2} \\
& \leq\left\|u_{n}-p\right\|^{2}-\left\|\Theta_{n}^{k} u_{n}-\Theta_{n}^{k-1} u_{n}\right\|^{2} \\
& \leq\left\|x_{n}-p\right\|^{2}-\left\|\Theta_{n}^{k} u_{n}-\Theta_{n}^{k-1} u_{n}\right\|^{2} .
\end{aligned}
$$

Set $\theta_{n}=\gamma f\left(x_{n}\right)-B S_{n} y_{n}$, and let $\lambda>0$ be a constant such that

$$
\lambda>\sup _{n, k}\left\{\left\|\theta_{n}\right\|,\left\|x_{k}-p\right\|\right\}
$$

Using Lemma 2.2 and noting that $\|\cdot\|^{2}$ is convex, we derive, from (3.20),

$$
\begin{aligned}
\left\|x_{n+1}-p\right\|^{2} & =\left\|\left(1-\beta_{n}\right)\left(S_{n} y_{n}-p\right)+\beta_{n}\left(x_{n}-p\right)+\epsilon_{n} \theta_{n}\right\|^{2} \\
& \leq\left\|\left(1-\beta_{n}\right)\left(S_{n} y_{n}-p\right)+\beta_{n}\left(x_{n}-p\right)\right\|^{2}+2 \epsilon_{n}\left\langle\theta_{n}, x_{n+1}-p\right\rangle \\
& \leq\left(1-\beta_{n}\right)\left\|S_{n} y_{n}-p\right\|^{2}+\beta_{n}\left\|x_{n}-p\right\|^{2}+2 \lambda^{2} \epsilon_{n} \\
& \leq\left(1-\beta_{n}\right)\left\|y_{n}-p\right\|^{2}+\beta_{n}\left\|x_{n}-p\right\|^{2}+2 \lambda^{2} \epsilon_{n} \\
& \leq\left(1-\beta_{n}\right)\left(\left\|x_{n}-p\right\|^{2}-\left\|\Theta_{n}^{k} u_{n}-\Theta_{n}^{k-1} u_{n}\right\|^{2}\right)+\beta_{n}\left\|x_{n}-p\right\|^{2}+2 \lambda^{2} \epsilon_{n} \\
& \leq\left\|x_{n}-p\right\|^{2}-\left(1-\beta_{n}\right)\left\|\Theta_{n}^{k} u_{n}-\Theta_{n}^{k-1} u_{n}\right\|^{2}+2 \lambda^{2} \epsilon_{n} .
\end{aligned}
$$

It follows, by Step 2 and condition (C1), that

$$
\begin{aligned}
\left\|\Theta_{n}^{k} u_{n}-\Theta_{n}^{k-1} u_{n}\right\|^{2} & \leq \frac{1}{1-\beta_{n}}\left(\left\|x_{n}-p\right\|^{2}-\left\|x_{n+1}-p\right\|^{2}+2 \lambda^{2} \epsilon_{n}\right) \\
& \leq \frac{1}{1-\beta_{n}}\left(2 \lambda\left\|x_{n}-x_{n+1}\right\|+2 \lambda^{2} \epsilon_{n}\right) \longrightarrow 0, \text { as } n \longrightarrow \infty
\end{aligned}
$$

Step 4. We will prove $\lim _{n \rightarrow \infty}\left\|u_{n}-x_{n}\right\|=0$.

We note from (3.1)

$$
\begin{aligned}
\left\|x_{n}-S_{n} y_{n}\right\| \leq & \left\|x_{n}-S_{n-1} y_{n-1}\right\|+\left\|S_{n-1} y_{n-1}-S_{n-1} y_{n}\right\|+\left\|S_{n-1} y_{n}-S_{n} y_{n}\right\| \\
\leq & \epsilon_{n-1}\left\|\gamma f\left(x_{n-1}\right)-B S_{n-1} y_{n-1}\right\|+\left\|y_{n-1}-y_{n}\right\| \\
& +\beta_{n}\left\|x_{n-1}-S_{n-1} y_{n-1}\right\|+\sup \left\{\left\|S_{n+1} z-S_{n} z\right\|: z \in\left\|y_{n}\right\|\right\} .
\end{aligned}
$$

Since $\epsilon_{n} \rightarrow 0, \beta_{n} \rightarrow 0$ as $n \rightarrow \infty, \lim _{n \rightarrow \infty}\left\|y_{n+1}-y_{n}\right\|=0$ and $\sup \left\{\left\|S_{n+1} z-S_{n} z\right\|: z \in\left\{y_{n}\right\}\right\} \rightarrow$ 0 , we get

$$
\left\|x_{n}-S_{n} y_{n}\right\| \longrightarrow 0 \text { as } n \longrightarrow \infty
$$


Let $v \in \Omega$. Since $u_{n}=T_{r_{n}} x_{n}$, it follows from Lemma 2.1 that

$$
\begin{aligned}
\left\|u_{n}-v\right\|^{2} & =\left\|T_{r_{n}} x_{n}-T_{r_{n}} v\right\|^{2} \leq\left\langle T_{r_{n}} x_{n}-T_{r_{n}} v, x_{n}-v\right\rangle=\left\langle u_{n}-v, x_{n}-v\right\rangle \\
& \leq \frac{1}{2}\left(\left\|u_{n}-v\right\|^{2}+\left\|x_{n}-v\right\|^{2}-\left\|u_{n}-x_{n}\right\|^{2}\right),
\end{aligned}
$$

and hence $\left\|u_{n}-v\right\|^{2} \leq\left\|x_{n}-v\right\|^{2}-\left\|u_{n}-x_{n}\right\|^{2}$. Therefore, using Lemma 2.6 and (3.22), we have

$$
\begin{aligned}
\left\|x_{n+1}-v\right\|^{2} & \leq\left(1-\beta_{n}\right)\left\|y_{n}-p\right\|^{2}+\beta_{n}\left\|x_{n}-p\right\|^{2}+2 \lambda^{2} \epsilon_{n} \\
& \leq\left(1-\beta_{n}\right)\left\|u_{n}-p\right\|^{2}+\beta_{n}\left\|x_{n}-p\right\|^{2}+2 \lambda^{2} \epsilon_{n} \\
& \leq\left(1-\beta_{n}\right)\left(\left\|x_{n}-v\right\|^{2}-\left\|u_{n}-x_{n}\right\|^{2}\right)+\beta_{n}\left\|x_{n}-p\right\|^{2}+2 \lambda^{2} \epsilon_{n} \\
& \leq\left\|x_{n}-v\right\|^{2}-\left(1-\beta_{n}\right)\left\|u_{n}-x_{n}\right\|^{2}+2 \lambda^{2} \epsilon_{n}
\end{aligned}
$$

and hence

$$
\begin{aligned}
\left\|u_{n}-x_{n}\right\|^{2} & \leq \frac{1}{1-\beta_{n}}\left(\left\|x_{n}-v\right\|^{2}-\left\|x_{n+1}-v\right\|^{2}+2 \lambda^{2} \epsilon_{n}\right) \\
& \leq \frac{1}{1-\beta_{n}}\left\{\left\|x_{n}-x_{n+1}\right\|\left(\left\|x_{n}-v\right\|-\left\|x_{n+1}-v\right\|\right)+2 \lambda^{2} \epsilon_{n}\right\} .
\end{aligned}
$$

Since $\left\{x_{n}\right\}$ is bounded, $\lim _{n \rightarrow \infty} \epsilon_{n}=0, \lim _{n \rightarrow \infty}\left\|x_{n}-x_{n+1}\right\|=0$, and $\lambda>\sup _{n, k}\left\{\left\|\theta_{n}\right\|,\left\|x_{k}-p\right\|\right\}$, it follows that

$$
\lim _{n \rightarrow \infty}\left\|x_{n}-u_{n}\right\|=0
$$

Next we will prove $\lim _{n \rightarrow \infty}\left\|u_{n}-y_{n}\right\|=0$.

$$
\begin{aligned}
\left\|u_{n}-y_{n}\right\|= & \left\|\Theta_{n}^{N} u_{n}-u_{n}\right\| \\
\leq & \left\|\Theta_{n}^{N} u_{n}-\Theta_{n}^{N-1} u_{n}\right\|+\left\|\Theta_{n}^{N-1} u_{n}-\Theta_{n}^{N-2} u_{n}\right\| \\
& +\cdots+\left\|\Theta_{n}^{2} u_{n}-\Theta_{n}^{1} u_{n}\right\|+\left\|\Theta_{n}^{1} u_{n}-\Theta_{n}^{0} u_{n}\right\|+\left\|u_{n}-u_{n}\right\| .
\end{aligned}
$$

From (2.10), we obtain

$$
\lim _{n \rightarrow \infty}\left\|u_{n}-y_{n}\right\|=0
$$

In addition, according to $\left\|x_{n}-y_{n}\right\| \leq\left\|x_{n}-u_{n}\right\|+\left\|u_{n}-y_{n}\right\|$, we have

$$
\lim _{n \rightarrow \infty}\left\|x_{n}-y_{n}\right\|=0
$$


It follows from (3.25), (3.32), and the inequality $\left\|y_{n}-S_{n} y_{n}\right\| \leq\left\|y_{n}-x_{n}\right\|+\left\|x_{n}-S_{n} y_{n}\right\|$ that $\lim _{n \rightarrow \infty}\left\|y_{n}-S_{n} y_{n}\right\|=0$. Since

$$
\begin{aligned}
\left\|S y_{n}-y_{n}\right\| & \leq\left\|S y_{n}-S_{n} y_{n}\right\|+\left\|S_{n} y_{n}-y_{n}\right\| \\
& \leq \sup \left\{\left\|S z-S_{n} z\right\|: z \in\left\{y_{n}\right\}\right\}+\left\|S_{n} y_{n}-y_{n}\right\|,
\end{aligned}
$$

for all $n \in \mathbb{N}$, it follows that

$$
\lim _{n \rightarrow \infty}\left\|S y_{n}-y_{n}\right\|=0
$$

Step 5. We show $\omega \in\left(\bigcap_{n=1}^{\infty} F_{i x}\left(S_{n}\right)\right) \cap \operatorname{MEP}\left(F_{1}, F_{2}\right) \cap\left(\bigcap_{i=1}^{N} I\left(A_{i}, M_{i}\right)\right)$.

Since $\left\{x_{n}\right\}$ is bounded, there exists a subsequence $\left\{x_{n_{i}}\right\}$ of $\left\{x_{n}\right\}$ which converges weakly to $\omega$. From (3.29), we obtain $\left\{u_{n_{i}}\right\}$ which converges weakly to $\omega$. From (3.32), it follows that $y_{n_{i}}-\omega$. We show $\omega \in \operatorname{MEP}\left(F_{1}, F_{2}\right)$. According to (3.1) and (A2), we obtain

$$
\frac{1}{r_{n}}\left\langle y-u_{n}, u_{n}-x_{n}\right\rangle \geq F_{1}\left(y, u_{n}\right)+F_{2}\left(y, u_{n}\right)
$$

and hence

$$
\left\langle y-u_{n_{i}}, \frac{u_{n_{i}}-x_{n_{i}}}{r_{n_{i}}}\right\rangle \geq F_{1}\left(y, u_{n}\right)+F_{2}\left(y, u_{n}\right)
$$

Since $\left(u_{n_{i}}-x_{n_{i}}\right) / r_{n_{i}} \rightarrow 0$ and $u_{n_{i}} \rightarrow \omega$, from (A4), it follows that $0 \geq F_{1}(y, \omega)+F_{2}(y, \omega)$ for all $y \in H$. For $t$ with $0<t \leq 1$ and $y \in H$, let $y_{t}=t y+(1-t) \omega$, then we obtain $0 \geq F_{1}\left(y_{t}, \omega\right)+F_{2}\left(y_{t}, \omega\right)$. So, from (A1) and (A4) we have

$$
\begin{aligned}
0 & =F_{1}\left(y_{t}, y_{t}\right)+F_{2}\left(y_{t}, y_{t}\right) \\
& \leq t F_{1}\left(y_{t}, y\right)+(1-t) F_{1}\left(y_{t}, \omega\right)+t F_{2}\left(y_{t}, y\right)+(1-t) F_{2}\left(y_{t}, \omega\right) \\
& \leq F_{1}\left(y_{t}, y\right)+F_{2}\left(y_{t}, y\right)
\end{aligned}
$$

and hence $0 \leq F_{1}\left(y_{t}, y\right)+F_{2}\left(y_{t}, y\right)$. From (A3), we have $0 \leq F_{1}(\omega, y)+F_{2}(\omega, y)$ for all $y \in H$. Therefore, $\omega \in \operatorname{MEP}\left(F_{1}, F_{2}\right)$.

Next, we show $\omega \in \bigcap_{n=1}^{\infty} F_{i x}\left(S_{n}\right)$. Assume $\omega \notin \bigcap_{n=1}^{\infty} F_{i x}\left(S_{n}\right)$; then we have $\omega \neq S \omega$. It follows by the Opial's condition and (3.34) that

$$
\begin{aligned}
\liminf _{n \rightarrow \infty}\left\|y_{n}-\omega\right\| & <\liminf _{n \rightarrow \infty}\left\|y_{n}-S \omega\right\| \\
& \leq \liminf _{n \rightarrow \infty}\left\{\left\|y_{n}-S y_{n}\right\|+\left\|S y_{n}-S \omega\right\|\right\} \\
& \leq \liminf _{n \rightarrow \infty}\left\|y_{n}-\omega\right\| .
\end{aligned}
$$

This is a contradiction. Hence $\omega \in \bigcap_{n=1}^{\infty} F_{i x}\left(S_{n}\right)$.

We now show that $\omega \in \bigcap_{i=1}^{N} I\left(A_{i}, M_{i}\right)$. In fact, since $A_{i}$ is $\alpha_{i}$-inverse-strongly monotone, $A_{i}, i=1,2, \ldots, N$, is a $1 / \alpha_{i}$-Lipschitz continuous monotone mapping and $D\left(A_{i}\right)=H$, 
Journal of Applied Mathematics

$i=1,2, \ldots, N$. It follows from Lemma 2.2 that $M_{i}+A_{i}, i=1,2, \ldots, N$, is maximal monotone. Let $(p, g) \in G\left(M_{i}+A_{i}\right), i=1,2, \ldots, N$, that is, $g-A_{i} p \in\left(M_{i} p\right), i=1,2, \ldots, N$. Since $\Theta_{n}^{k} u_{n}=J_{M_{k}, \lambda_{k, n}}\left(I-\lambda_{k, n} A_{k}\right) \Theta_{n}^{k-1} u_{n}$, we have $\Theta_{n}^{k-1} u_{n}-\lambda_{k, n} A_{k} \Theta_{n}^{k-1} u_{n} \in\left(I+\lambda_{k, n} M_{k}\right)\left(\Theta_{n}^{k} u_{n}\right)$, that is,

$$
\frac{1}{\lambda_{n, k}}\left(\Theta_{n}^{k-1} u_{n}-\Theta_{n}^{k} u_{n}-\lambda_{N, n} A_{k} \Theta_{n}^{k-1} u_{n}\right) \in M_{k}\left(\Theta_{n}^{k} u_{n}\right)
$$

By the maximal monotonicity of $M_{i}+A_{i} i=1,2, \ldots, N$, we have

$$
\left\langle p-\Theta_{n}^{k} u_{n}, g-A_{k} p-\frac{1}{\lambda_{k, n}}\left(\Theta_{n}^{k-1} u_{n}-\Theta_{n}^{k} u_{n}-\lambda_{k, n} A_{k} \Theta_{n}^{k-1} u_{n}\right)\right\rangle \geq 0,
$$

which implies

$$
\begin{aligned}
\left\langle p-\Theta_{n}^{k} u_{n}, g\right\rangle & \geq\left\langle p-\Theta_{n}^{k} u_{n}, A_{k} p+\frac{1}{\lambda_{n, k}}\left(\Theta_{n}^{k-1} u_{n}-\Theta_{n}^{k} u_{n}-\lambda_{k, n} A_{k} \Theta_{n}^{k-1} u_{n}\right)\right\rangle \\
& =\left\langle p-\Theta_{n}^{k} u_{n}, A_{k} p-A_{k} \Theta_{n}^{k} u_{n}+A_{k} \Theta_{n}^{k} u_{n}-A_{k} \Theta_{n}^{k-1} u_{n}+\frac{1}{\lambda_{k, n}}\left(\Theta_{n}^{k-1} u_{n}-\Theta_{n}^{k} u_{n}\right)\right\rangle \\
& \geq 0+\left\langle p-\Theta_{n}^{k} u_{n}, A_{k} \Theta_{n}^{k} u_{n}-A_{k} \Theta_{n}^{k-1} u_{n}\right\rangle+\left\langle p-\Theta_{n}^{k} u_{n}, \frac{1}{\lambda_{k, n}}\left(\Theta_{n}^{k-1} u_{n}-\Theta_{n}^{k} u_{n}\right)\right\rangle,
\end{aligned}
$$

for $k \in\{1,2, \ldots, N\}$. From (3.17), it follows $\lim _{n \rightarrow \infty}\left\|\Theta_{n}^{k} u_{n}-\Theta_{n}^{k-1} u_{n}\right\|=0$, especially, $\Theta_{n_{i}}^{k} u_{n_{i}} \rightarrow \omega$. Since $A_{k}, k=1, \ldots, N$, are Lipschitz continuous operators, we have $\| A_{k} \Theta_{n}^{k-1} u_{n}-$ $A_{k} \Theta_{n}^{k} u_{n} \| \rightarrow 0$. So, from (3.41), we have

$$
\lim _{i \rightarrow \infty}\left\langle p-\Theta_{n_{i}}^{k} u_{n_{i}}, g\right\rangle=\langle p-\omega, g\rangle \geq 0
$$

Since $A_{k}+M_{k}, k \in\{1,2, \ldots, N\}$ is maximal monotone, this implies that $0 \in\left(M_{k}+A_{k}\right)(\omega)$, $k \in\{1,2, \ldots, N\}$, that is, $\omega \in \bigcap_{i=1}^{N} I\left(A_{i}, M_{i}\right)$. So, we obtain result.

Step 6. We show that

$$
\limsup _{n \rightarrow \infty}\left\langle(B-\gamma f) z, z-x_{n}\right\rangle \leq 0
$$

where $z=P_{\Omega}(I-B+\gamma f)(z)$ is unique solution of the variational inequality (3.2).

To show this, we choose a subsequence $\left\{x_{n_{i}}\right\}$ of $\left\{x_{n}\right\}$ such that

$$
\lim _{i \rightarrow \infty}\left\langle(B-\gamma f) z, z-x_{n_{i}}\right\rangle=\limsup _{n \rightarrow \infty}\left\langle(B-\gamma f) z, z-x_{n}\right\rangle .
$$

By the proof of Step 5, we obtain that

$$
\limsup _{n \rightarrow \infty}\left\langle(B-\gamma f) z, z-x_{n}\right\rangle=\lim _{i \rightarrow \infty}\left\langle(B-\gamma f) z, z-x_{n_{i}}\right\rangle=\langle(B-\gamma f) z, z-\omega\rangle \leq 0 .
$$


Step 7. We prove that $x_{n} \rightarrow \omega$.

By using Lemmas 2.3 and 2.4, we have

$$
\begin{aligned}
\left\|x_{n+1}-\omega\right\|^{2}= & \left\|\left(\left(1-\beta_{n}\right) I-\epsilon_{n} B\right)\left(S_{n} y_{n}-\omega\right)+\beta_{n}\left(x_{n}-\omega\right)+\epsilon_{n}\left(\gamma f\left(x_{n}\right)-B \omega\right)\right\|^{2} \\
\leq & \left\|\left(\left(1-\beta_{n}\right) I-\epsilon_{n} B\right)\left(S_{n} y_{n}-\omega\right)+\beta_{n}\left(x_{n}-\omega\right)\right\|^{2}+2 \epsilon_{n}\left\langle\gamma f\left(x_{n}\right)-B \omega, x_{n+1}-\omega\right\rangle \\
\leq & \left.\|\left(1-\beta_{n}\right) I-\epsilon_{n} B\right)\left(S_{n} y_{n}-\omega\right)\left\|^{2}+\beta_{n}\right\| x_{n}-\omega \|^{2} \\
& +2 \epsilon_{n}\left\langle\gamma f\left(x_{n}\right)-f(\omega), x_{n+1}-\omega\right\rangle+2 \epsilon_{n}\left\langle\gamma f(\omega)-B \omega, x_{n+1}-\omega\right\rangle \\
\leq & \left(\left(1-\beta_{n}\right)-\epsilon_{n} \bar{\gamma}\right)\left\|S_{n} y_{n}-\omega\right\|^{2}+\beta_{n}\left\|x_{n}-\omega\right\|^{2} \\
& +2 \epsilon_{n} \gamma \alpha\left\|x_{n}-\omega\right\|\left\|x_{n+1}-\omega\right\|+2 \epsilon_{n}\left\langle\gamma f(\omega)-B \omega, x_{n+1}-\omega\right\rangle \\
\leq & \left(\left(1-\beta_{n}\right)-\epsilon_{n} \bar{\gamma}\right)\left\|x_{n}-\omega\right\|^{2}+\beta_{n}\left\|x_{n}-\omega\right\|^{2} \\
& +\epsilon_{n} \gamma \alpha\left(\left\|x_{n}-\omega\right\|^{2}+\left\|x_{n+1}-\omega\right\|^{2}\right)+2 \epsilon_{n}\left\langle\gamma f(\omega)-B \omega, x_{n+1}-\omega\right\rangle \\
\leq & \left(1-\epsilon_{n}(\bar{\gamma}-\gamma \alpha)\right)\left\|x_{n}-\omega\right\|^{2}+\epsilon_{n} \gamma \alpha\left\|x_{n+1}-\omega\right\|^{2}+2 \epsilon_{n}\left\langle\gamma f(\omega)-B \omega, x_{n+1}-\omega\right\rangle .
\end{aligned}
$$

It follows that

$$
\left\|x_{n+1}-\omega\right\|^{2} \leq\left(1-\frac{(\bar{\gamma}-\alpha \gamma) \epsilon_{n}}{1-\alpha \gamma \epsilon_{n}}\right)\left\|x_{n}-\omega\right\|^{2}+\frac{2 \epsilon_{n}}{1-\alpha \gamma \epsilon_{n}}\left\langle\gamma f(\omega)-B \omega, x_{n+1}-\omega\right\rangle .
$$

Now, from conditions (C1), (C2), and (C6), Step 6 and Lemma 2.8, we obtain $\lim _{n \rightarrow \infty} \| x_{n}-$ $\omega \|=0$. Namely, $x_{n} \rightarrow \omega$ in norm.

Corollary 3.2. Let $H$ be a real Hilbert space, let $F$ be a bifunction $H \times H \rightarrow \mathbb{R}$ satisfying (A1)$(A 4)$, and let $\left\{S_{n}\right\}$ be a sequence of nonexpansive mappings on $H$. Let $A_{i}: H \rightarrow H, i=1,2, \ldots, N$ be $\alpha_{i}$-inverse-strongly monotone mappings and $M_{i}: H \rightarrow 2^{H}, i=1,2, \ldots, N$, maximal monotone mappings such that $\Omega:=\left(\bigcap_{n=1}^{\infty} F_{i x}\left(S_{n}\right)\right) \cap E P(F) \cap\left(\bigcap_{i=1}^{N} I\left(A_{i}, M_{i}\right)\right) \neq \emptyset$. Let $f$ be a contraction of $H$ into itself with a constant $\alpha \in(0,1)$, and let $B$ be a strongly positive bounded linear operator on $H$ with coefficient $\bar{\gamma}>0$ and $0<\gamma<\bar{\gamma} / \alpha$. Let $\left\{x_{n}\right\},\left\{y_{n}\right\}$, and $\left\{u_{n}\right\}$ be sequences generated by $x_{1} \in H$ and

$$
\begin{gathered}
F\left(u_{n}, y\right)+\frac{1}{r_{n}}\left\langle y-u_{n}, u_{n}-x_{n}\right\rangle \geq 0, \quad \forall y \in H, \\
y_{n}=J_{M_{N}, \lambda_{N, n}}\left(I-\lambda_{N, n} A_{N}\right) \cdots J_{M_{1}, \lambda_{1, n}}\left(I-\lambda_{1, n} A_{1}\right) u_{n}, \\
x_{n+1}=\epsilon_{n} \gamma f\left(x_{n}\right)+\beta_{n} x_{n}+\left(\left(1-\beta_{n}\right) I-\epsilon_{n} B\right) S_{n} y_{n},
\end{gathered}
$$

for all $n \in \mathbb{N}$, where $\beta_{n} \in(0,1) \lambda_{i, n} \in\left(0,2 \alpha_{i}\right], i \in\{1,2, \ldots, N\}$, satisfy (H1)-(H2) and $\left\{\epsilon_{n}\right\} \subset[0,1]$, and $\left\{r_{n}\right\} \subset(0, \infty)$ satisfy

(C1) $\lim _{n \rightarrow \infty} \epsilon_{n}=0$;

(C2) $\sum_{n=1}^{\infty} \epsilon_{n}=\infty$;

(C3) $\sum_{n=1}^{\infty}\left|\epsilon_{n+1}-\epsilon_{n}\right|<\infty$; 
(C4) $\lim \inf _{n \rightarrow \infty} r_{n}>0$;

(C5) $\sum_{n=1}^{\infty}\left|r_{n+1}-r_{n}\right|<\infty$;

(C6) $\lim _{n \rightarrow \infty} \beta_{n}=0$.

Suppose that $\sum_{n=1}^{\infty} \sup \left\{\left\|S_{n+1} z-S_{n} z\right\|: z \in K\right\}<\infty$ for any bounded subset $K$ of $H$. Let $S$ be a mapping of $H$ into itself defined by $S x=\lim _{n \rightarrow \infty} S_{n} x$, for all $x \in H$, and suppose that $F_{i x}(S)=$ $\bigcap_{n=1}^{\infty} F_{i x}\left(S_{n}\right)$. Then, $\left\{x_{n}\right\},\left\{y_{n}\right\}$, and $\left\{u_{n}\right\}$ converge strongly to $z$, where $z=P_{\Omega}(I-B+\gamma f)(z)$ is a unique solution of the variational inequality

$$
\langle(B-\gamma f) z, z-x\rangle \leq 0 \quad x \in \Omega .
$$

\section{Acknowledgment}

This work is supported in part by China Postdoctoral Science Foundation (Grant no. 20100470783).

\section{References}

[1] P. L. Combettes and S. A. Hirstoaga, "Equilibrium programming in Hilbert spaces," Journal of Nonlinear and Convex Analysis, vol. 6, no. 1, pp. 117-136, 2005.

[2] S. Plubtieng and R. Punpaeng, "A new iterative method for equilibrium problems and fixed point problems of nonexpansive mappings and monotone mappings," Applied Mathematics and Computation, vol. 197, no. 2, pp. 548-558, 2008.

[3] L. C. Zeng, S. Schaible, and J. C. Yao, "Iterative algorithm for generalized set-valued strongly nonlinear mixed variational-like inequalities," Journal of Optimization Theory and Applications, vol. 124 no. 3, pp. 725-738, 2005.

[4] S. S. Chang, H. W. Joseph Lee, and C. K. Chan, "A new method for solving equilibrium problem fixed point problem and variational inequality problem with application to optimization," Nonlinear Analysis: Theory, Methods \& Applications, vol. 70, no. 9, pp. 3307-3319, 2009.

[5] V. Colao, G. Marino, and H.-K. Xu, "An iterative method for finding common solutions of equilibrium and fixed point problems," Journal of Mathematical Analysis and Applications, vol. 344, no. 1, pp. 340 352, 2008.

[6] S. Plubtieng and R. Punpaeng, "A general iterative method for equilibrium problems and fixed point problems in Hilbert spaces," Journal of Mathematical Analysis and Applications, vol. 336, no. 1, pp. 455$469,2007$.

[7] R. T. Rockafellar, "Monotone operators and the proximal point algorithm," SIAM Journal on Control and Optimization, vol. 14, no. 5, pp. 877-898, 1976.

[8] S. Adly, "Perturbed algorithms and sensitivity analysis for a general class of variational inclusions," Journal of Mathematical Analysis and Applications, vol. 201, no. 2, pp. 609-630, 1996.

[9] H. H. Bauschke and J. M. Borwein, "On projection algorithms for solving convex feasibility problems," SIAM Review, vol. 38, no. 3, pp. 367-426, 1996.

[10] S. Plubtieng and W. Sriprad, "A viscosity approximation method for finding common solutions of variational inclusions, equilibrium problems, and fixed point problems in Hilbert spaces," Fixed Point Theory and Applications, vol. 2009, Article ID 567147, 20 pages, 2009.

[11] Y. Yao, Y. J. Cho, and Y.-C. Liou, "Algorithms of common solutions for variational inclusions, mixed equilibrium problems and fixed point problems," European Journal of Operational Research, vol. 212, no. 2, pp. 242-250, 2011.

[12] S. Saeidi, "Iterative algorithms for finding common solutions of variational inequalities and systems of equilibrium problems and fixed points of families and semigroups of nonexpansive mappings," Nonlinear Analysis: Theory, Methods E Applications, vol. 70, no. 12, pp. 4195-4208, 2009.

[13] K. Aoyama, Y. Kimura, W. Takahashi, and M. Toyoda, “Approximation of common fixed points of a countable family of nonexpansive mappings in a Banach space," Nonlinear Analysis: Theory, Methods $\mathcal{E}$ Applications, vol. 67, no. 8, pp. 2350-2360, 2007. 
[14] J. W. Peng, Y. Wang, D. S. Shyu, and J. C. Yao, "Common solutions of an iterative scheme for variational inclusions, equilibrium problems, and fixed point problems," Journal of Inequalities and Applications, vol. 2008, Article ID 720371, 15 pages, 2008.

[15] F. Cianciaruso, G. Marino, L. Muglia, and Y. Hong, "A hybrid projection algorithm for finding solutions of mixed equilibium problem and variational inequality problem," Fixed Point Theory and Applications, vol. 2010, Article ID 383740, 19 pages, 2010.

[16] H. Brézis, Opérateurs Maximaux Monotones et Semi-Groupes de Contractions dans les Es-paces de Hilbert, North-Holland, Amsterdam, The Netherlands, 1973.

[17] B. Lemaire, "Which fixed point does the iteration method select?" in Recent Advances in Optimization (Trier, 1996), vol. 452 of Lecture Notes in Economics and Mathematical Systems, pp. 154-167, Springer, Berlin, Germany, 1997.

[18] G. Marino and H.-K. Xu, "A general iterative method for nonexpansive mappings in Hilbert spaces," Journal of Mathematical Analysis and Applications, vol. 318, no. 1, pp. 43-52, 2006.

[19] T. Suzuki, "Strong convergence of Krasnoselskii and Mann's type sequences for one-parameter nonexpansive semigroups without Bochner integrals," Journal of Mathematical Analysis and Applications, vol. 305, no. 1, pp. 227-239, 2005.

[20] H.-K. Xu, "Iterative algorithms for nonlinear operators," Journal of the London Mathematical Society. Second Series, vol. 66, no. 1, pp. 240-256, 2002. 


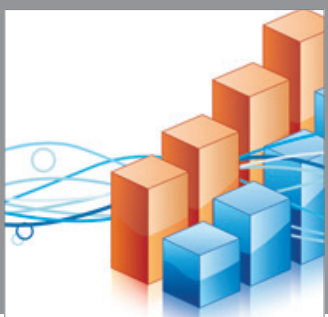

Advances in

Operations Research

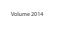

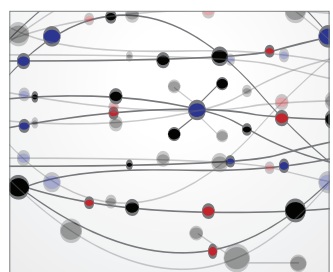

\section{The Scientific} World Journal
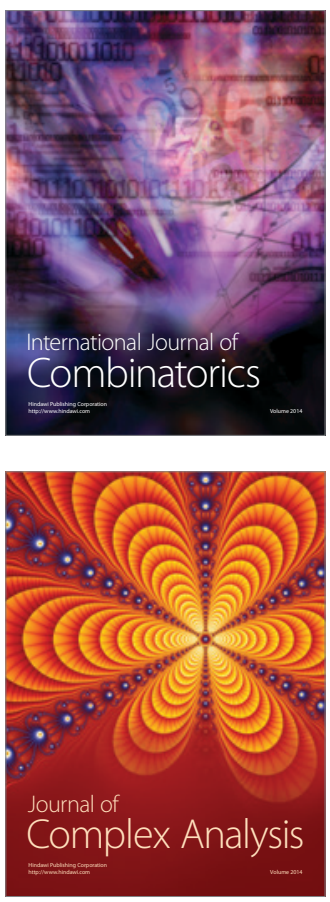

International Journal of

Mathematics and

Mathematical

Sciences
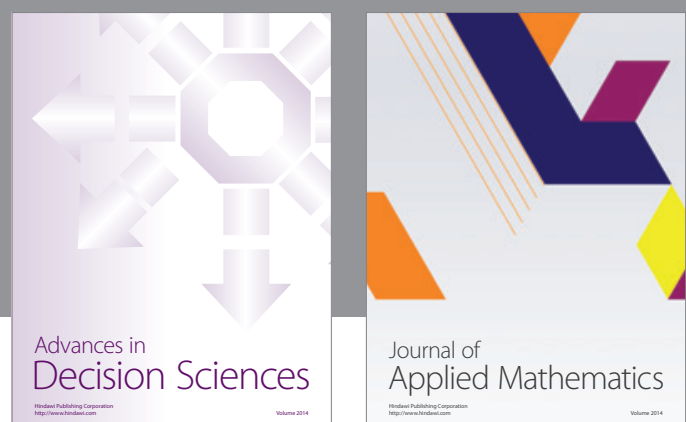

Journal of

Applied Mathematics
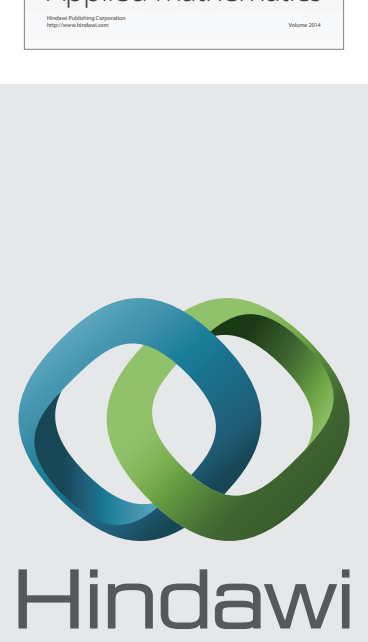

Submit your manuscripts at http://www.hindawi.com
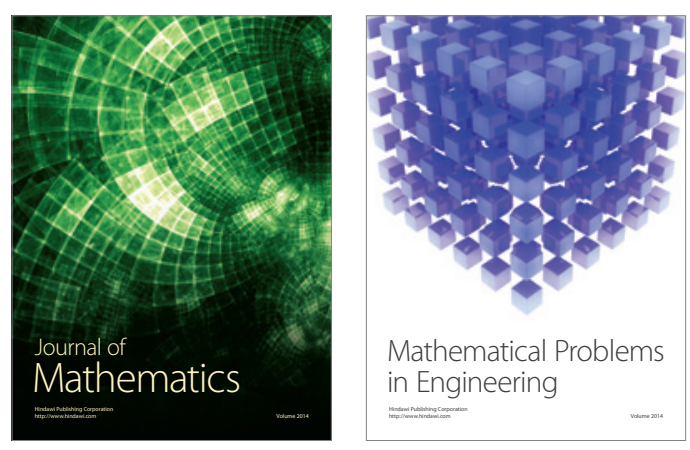

Mathematical Problems in Engineering
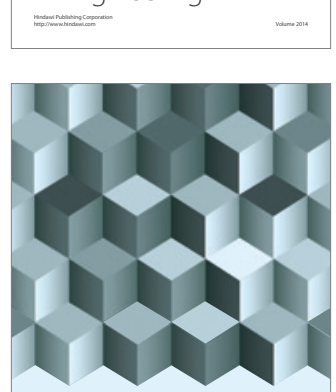

Journal of

Function Spaces
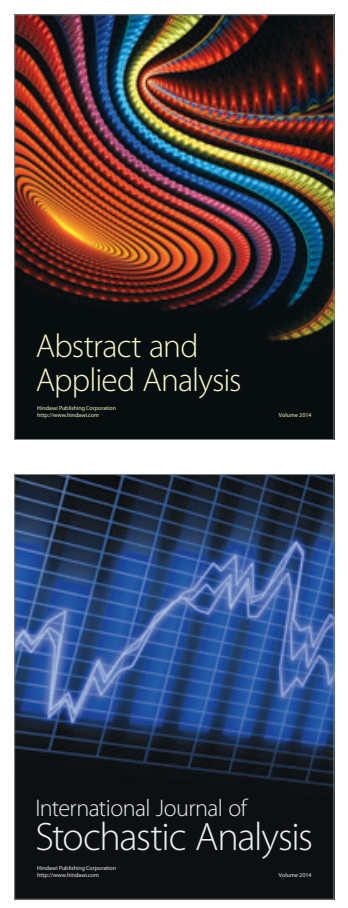

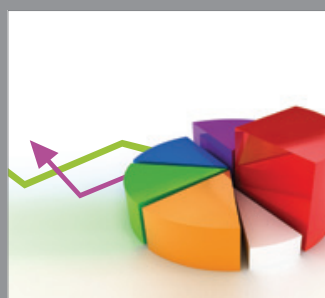

ournal of

Probability and Statistics

Promensencen
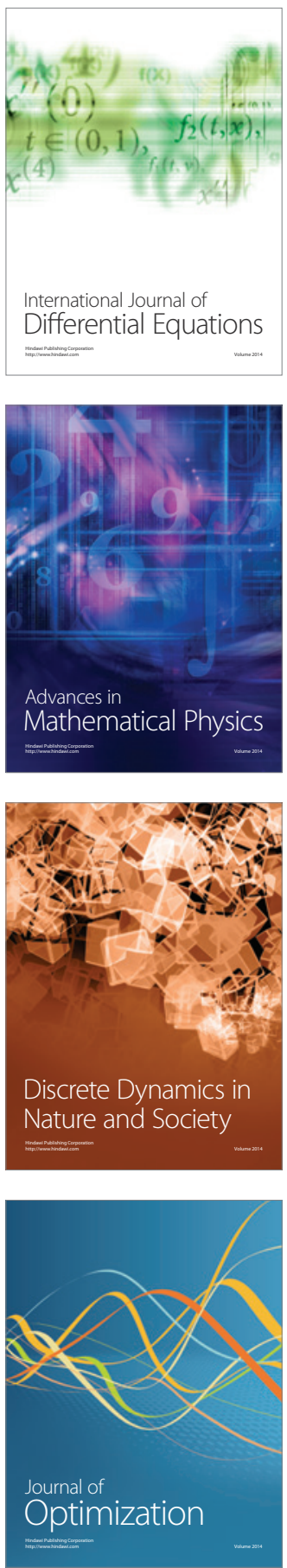